\title{
Characterization of Deformation Behaviors and Elastic Moduli of Multilayered Films in Piezoelectric Inkjet Head
}

\author{
Seong-Gu Hong, Minho Kim, Soon-Bok Lee, Member, IEEE, Member, ASME, and Chong Soo Lee
}

\begin{abstract}
A bulge testing system was developed to mechanically characterize the deformation behaviors and elastic moduli of multilayered films, mainly composed of polycrystalline silicon (polysilicon) and lead zirconate titanate (PZT), used in a multilayer actuator of a piezoelectric inkjet head. In the tests, commercial inkjet heads including a few tens of multilayer actuators were directly pressurized by air, and the corresponding deflections were measured via full-field optical measurement techniques. An analytic solution derived from a thin-plate theory and finite-element analysis were used to describe pressure-deflection behaviors of films, and the results were compared with the experimental data to evaluate the elastic modulus of individual film. The results showed that the elastic moduli of polysilicon and PZT films are $\sim 110$ and $\sim 49 \mathrm{GPa}$, respectively. These values were consistent with the nanoindentation results. For polysilicon films, about $30 \%$ reduction in elastic modulus, compared with that calculated from single-crystal elastic constants, was observed, and this was most likely attributed to the presence of microdefects like voids and microcracks at grain boundaries between columnar grains.

[2008-0056]
\end{abstract}

Index Terms-Bulge test, full-field optical measurement, multilayered films, piezoelectric inkjet head.

\section{INTRODUCTION}

W ITH THE rapid development of the drop-on-demand (DOD) inkjet printhead, there is a growing demand of using inkjet printing technology not only in office printing applications but also in promising industrial applications [1]. Piezoelectric inkjet printhead is a preferred choice for DOD industrial printing applications because it can handle a variety of jetting fluids and provide consistent high performance under harsh working conditions [2]; this technology uses electrical signals to change the shape of piezo elements and then fires ink droplets according to the physical force generated by the change in shape of these elements. It can provide the advantage of noncontact deposition and is already being utilized effectively

Manuscript received March 6, 2008; revised May 28, 2008 and July 31, 2008. First published September 16, 2008; current version published October 1, 2008. This work was supported by the National Research Laboratory Program of the Korean Ministry of Science and Technology under Grant ROA-2003000-10309-0. Subject Editor M. Spearing.

S.-G. Hong and C. S. Lee are with the Department of Materials Science and Engineering, Pohang University of Science and Technology, Pohang 790-784, Korea (e-mail: sghong@ postech.ac.kr; hsg@kaist.ac.kr; cslee@ postech.ac.kr).

M. Kim and S.-B. Lee are with the Department of Mechanical Engineering, Korea Advanced Institute of Science and Technology, Daejeon 305-701, Korea (e-mail: jjclassic@kaist.ac.kr; sblee@kaist.ac.kr).

Color versions of one or more of the figures in this paper are available online at http://ieeexplore.ieee.org.

Digital Object Identifier 10.1109/JMEMS.2008.2004795 in manufacturing processes of liquid crystal display, polyimide alignment layer, chromatic filter, fuel injector of engine, optical interconnects, organic light-emitting devices, etc. [1]-[4]. In most applications, it is desirable to provide superior ink ejection performance, compatibility with a wide variety of inks, and durability. Considerable efforts to achieve these goals have been made by optimizing the printhead structure via computer simulation, including both structural and electrical field analyses [2]-[4]; it is difficult to experimentally characterize the printhead because of its microscopic structure. Hence, a choice of reliable material properties for the printhead constituents is considered crucial in the design and development of the printhead, and the development of appropriate methodologies to characterize their material properties is attracting interest.

A piezoelectric inkjet head generally includes a plurality of multilayer actuators, which are made of layers of ceramic and piezoelectric crystals, ink chambers coupled to the multilayer actuators, and a nozzle plate of which nozzles are connected to individual ink chamber (refer to Fig. 1). Among these, the multilayer actuator is considered to be a core technology constituent as its size and performance directly influence the printhead design. As shown in Fig. 1(b), it is composed of multilayered films with a thickness in microscale. This microscopic feature makes it difficult to experimentally characterize the film properties used, particularly mechanical properties, and thus, in most studies, bulk properties have been used inevitably. It is known, however, that the electrical and mechanical properties of microscale films are strongly affected by the nature of their underlying microstructure, which is governed by processing conditions [5]-[10]. Therefore, it is recommended that material characterization should be conducted on specimens of similar sizes produced by the same methods to assure that the properties of the as-manufactured material are being measured, but the best way will be testing a real structure of interest directly.

In this paper, we developed a methodology to mechanically characterize the elastic behaviors of multilayered films used in a multilayer actuator. In piezoelectric inkjet heads, when an electric pulse is applied to the piezo element placed on the wall of the ink chamber in a multilayer actuator, the vibrating plate deforms to create a pressure wave that causes the ink droplet to be ejected through the nozzle. This operating mechanism makes it difficult to devise a testing method and also to analyze the test result not only because the deflection measurement of the concerned layer is nearly impossible due to the geometry but also because it includes the piezoelectric 


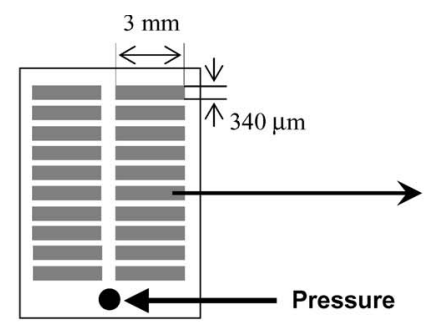

$<$ Top view of piezoelectric inkjet head $>$

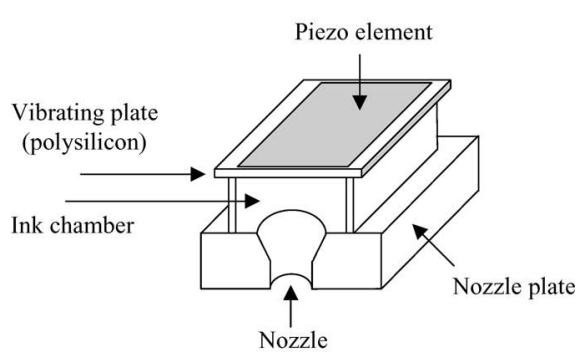

$<$ A multilayer actuator $>$

(a)

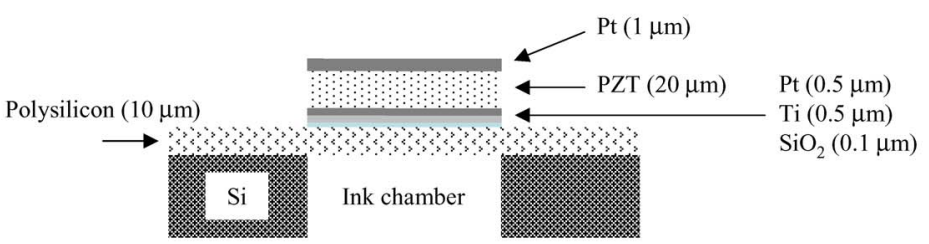

(b)

Fig. 1. Schematic drawings of (a) a piezoelectric inkjet head and (b) a cross section of a multilayer actuator.

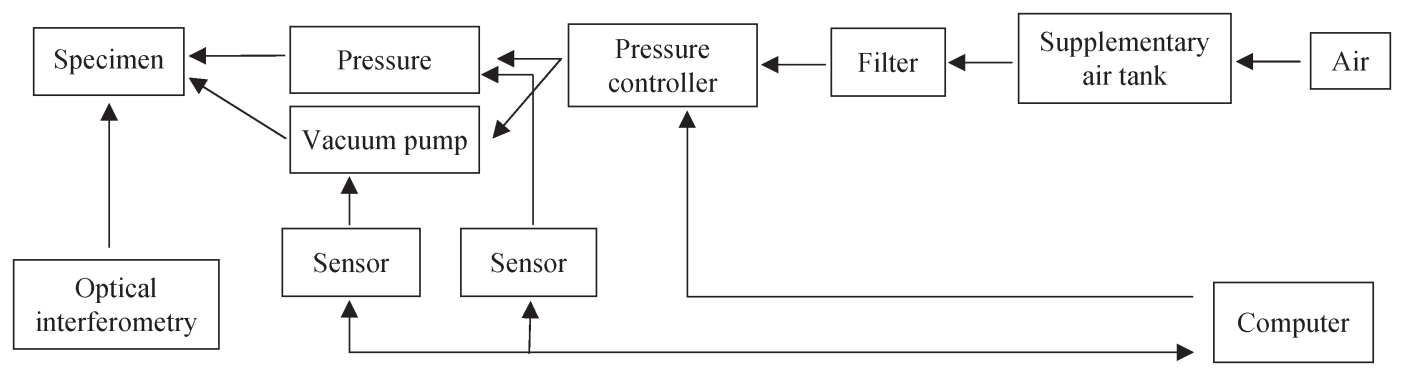

Fig. 2. Block diagram of the bulge test system.

effect. In this paper, however, our concern is to mechanically characterize the deformation behavior of the multilayer actuator and to evaluate the elastic moduli of multilayered films in it. A simple testing scheme can be applicable for this purpose without changing the actuator geometry; all nozzles in the inkjet head are blocked, and the pressure is applied through the flow channel connected to ink chambers. As the pressure is applied, the films bend in the upward direction, and this facilitates the deflection measurement. In the developed testing system, fullfield optical measurement techniques were adopted to measure the deflections of bulged films, and the results were compared with the predictions from a thin-plate theory and finite-element (FE) analysis to evaluate the elastic modulus of each layer.

\section{EXPERIMENT}

\section{A. Test Sample}

The test samples used in this paper were siliconmicromachined piezo inkjet printheads fabricated by the Micro System Laboratory of Samsung Advanced Institute of Technology, using standard micromachining techniques. The inkjet head is composed of the following two main plates: an upper plate including multilayer actuators and ink chambers, and a lower plate including a nozzle plate, nozzles, and flow channels. These two plates are connected together via eutectic bonding technique for completion. As shown in Fig. 1(b), the multilayer actuator consists of multilayer structure of polysilicon, silicon dioxide, bottom electrode of platinum and titanium thin films, piezoelectric layer of sol-gel lead zirconate titanate (PZT), and top electrode of platinum thin film; polysilicon film was deposited by the low-pressure chemical vapor deposition (LPCVD) process at $620{ }^{\circ} \mathrm{C}$ with a pressure of 200 mtorr. Specimens for polysilicon included just a polysilicon layer, and for PZT specimens, electrode layers were not deposited to rule out their effect on the test result. The dimensions of polysilicon and PZT films were $340 \times 3000 \times 10 \mu \mathrm{m}^{3}$ (width $\times$ length $\times$ thickness) and $\sim 340 \times 3000 \times \sim 20 \mu \mathrm{m}^{3}$, respectively (Fig. 1).

\section{B. Bulge Testing}

A block diagram of the developed bulge test system is shown in Fig. 2. Air was used as a pressurization medium, and pressure available ranged from -100 to $600 \mathrm{kPa}$. A supplementary air tank was used to regulate the pressure in each ink chamber because during the testing, an abrupt and unexpected pressure drop can occur by the failure of multilayer actuators. The corresponding displacement was measured with full-field optical interferometers, depending on the optical surface conditions of the specimens tested. The test was conducted under open-circuit condition.

Consider a pressurized rectangular plate made out of an isotropic elastic material. From Kirchhoff's plate theory [11], 


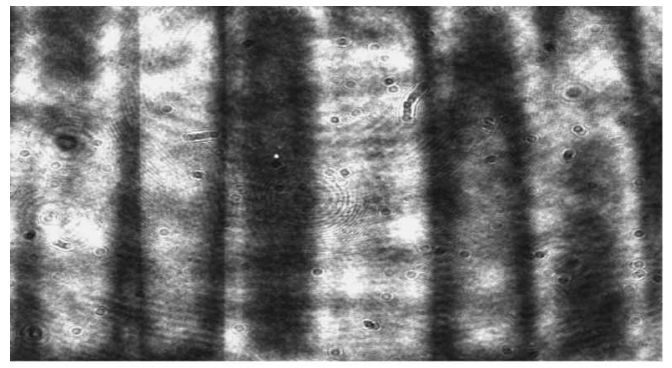

(a)

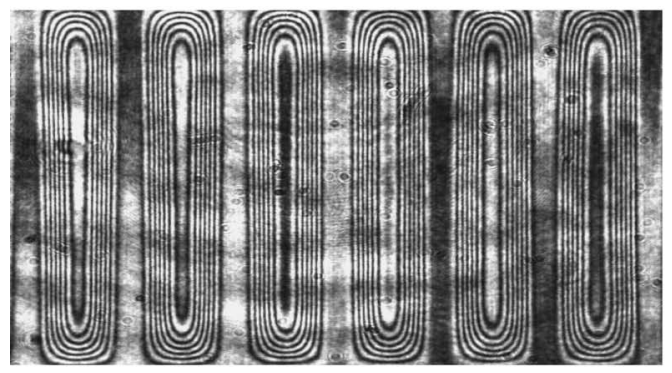

(c)

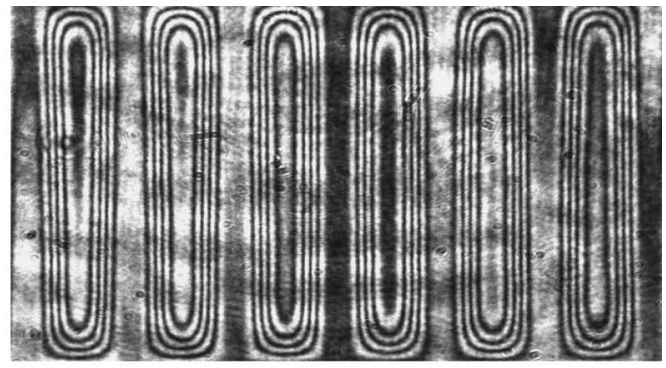

(b)

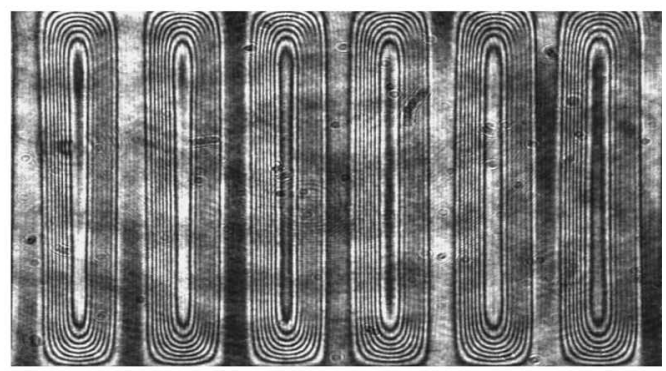

(d)

Fig. 3. Fringe pattern of the deflection contours of polysilicon films obtained by the Twyman-Green interferometry. (a) $0 \mathrm{kPa}$. (b) $222 \mathrm{kPa}$. (c) $400 \mathrm{kPa}$. (d) $575 \mathrm{kPa}$.

with the assumptions that: 1$)$ the deflection is small in comparison with the plate thickness $(h / t<0.3$, where $h$ and $t$ represent the maximum deflection and the plate thickness, respectively); 2) the plane strain condition holds along the length direction around the middle section, which can be achieved with the aspect ratio (the length-to-width ratio) greater than four [12]; 3 ) the plate thickness and the pressure applied are uniform over the whole plate; and 4) the boundary conditions are four clamped edges (i.e., $w(0)=w(a)=0$ and $w^{\prime}(0)=w^{\prime}(a)=$ $0)$, an analytic solution for the surface deflection can derived as

$$
w(x)=\frac{\left(1-\nu^{2}\right) p}{2 E t^{3}}\left(x^{4}-2 a x^{3}+a^{2} x^{2}\right)
$$

where $x$ is the coordinate along the plate width direction, $\nu$ is the Poisson's ratio, $E$ is the elastic modulus, $a$ is the plate width, $p$ is the pressure applied, and $w(x)$ is the deflection at the point $x$. The maximum deflection $h$ takes place at the center of the plate

$$
h=w\left(\frac{a}{2}\right)=\frac{\left(1-\nu^{2}\right) a^{4} p}{32 E t^{3}} .
$$

\section{Full-Field Optical Measurement Technique}

Polysilicon and PZT films have optically different surface conditions, namely, a specular surface for polysilicon and a nonmirrored surface for PZT. This fact restricts optical measurement techniques available. In this paper, two types of optical methods, i.e., Twyman-Green interferometry for polysilicon and electronic speckle pattern interferometry (ESPI) for PZT, were adopted to measure out-of-plane displacements during bulge testing. The detailed discussion on the optical techniques available for measuring out-of-plane displacements can be found elsewhere [13].
The Twyman-Green interferometry was constructed by following the concept suggested by Post et al. [14], where a 632.8-nm-wavelength He-Ne laser is used with spatial filter and collimating lens to realize collimating beam with $30.0-\mathrm{mm}$ diameter. The basic sensitivity was $316.5 \mathrm{~nm} /$ fringe, and the improvement in sensitivity up to $50 \mathrm{~nm} /$ fringe could be achieved by adopting a phase-shifting method, which was embodied by mounting the PZT actuating stage at the reference mirror stage. In addition, an image processing filter [15] was employed to improve the fringe image that may contain many noises. The fringe analysis was conducted with a free software, i.e., IDEA [16].

For the ESPI system, a commercial one (Dantec-Ettemeyer Q-300) and image data processing software supplied by Ettemeyer (ISTRA, version 3.3) were used. The system provides the sensitivity up to $23 \mathrm{~nm} /$ fringe by phase shifting the beam splitter and includes effective filtering and smoothing tools that cope with imperfections and low- and high-frequency noises. A comparative test was preliminarily carried out to resolve some reduction in accuracy which may be introduced by the following reasons. 1) Image processing schemes such as smoothing and filtering may affect the deflection field calculated. 2) The software, ISTRA, is required to define the distance between the specimen and the camera's reference plane, and the uncertainty in this value will distort the data. In the comparative test, the deflection of polysilicon specimen was measured with both ESPI and Twyman-Green interferometry; for ESPI, diffusive paint with a particle size of about the wavelength of the laser light employed was sprayed on the specimen surface to make it nonmirrored. It was found that the effect of image processing schemes is negligible, and the error, which originated from the uncertainty in defining the distance between the specimen and the camera's reference plane, can be fixed by finding a correction factor correlating the maximum deflection-pressure data obtained by both optical measurement techniques. 


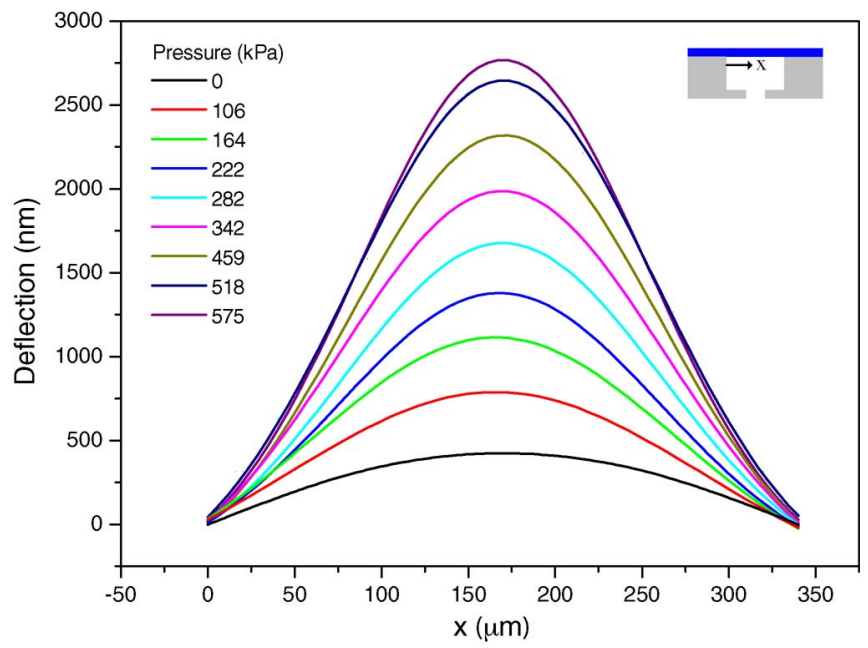

Fig. 4. Transverse deflection profiles at the middle section of the third polysilicon film obtained by the Twyman-Green interferometry.

\section{RESUlts AND Discussion}

\section{A. Polysilicon Film}

The fringe patterns of deflection contours of six polysilicon films with the pressure are shown in Fig. 3, where each fringe interval corresponds to $316.5 \mathrm{~nm}$. Fig. 4 shows the transverse deflection profiles at the middle section of the third polysilicon film. The maximum deflection takes place at the center of the film and is about $2700 \mathrm{~nm}$ under the maximum pressure of $575 \mathrm{kPa}$. In Fig. 4, the maximum initial deflection of $\sim 400 \mathrm{~nm}$, which was induced by the fabrication process of the specimen, can be noted. This initial deflection should be properly considered when comparing the experimental results with the results from the analytic solution and the FE analysis. The principle of superposition can be available for this purpose [17]. The principle describes that if the displacement gradient is infinitesimal and the material deforms elastically, then a linear theory is available and the principle holds. Obeying the principle, only the deflection induced by the pressure applied can be taken into account in the analysis of the testing results with the analytic solution and the FE analysis; the pressureinduced deflection can be obtained by subtracting the initial deflection from the value measured by the Twyman-Green interferometry.

Since the deflection-to-thickness ratio is $<0.3$, the aspect ratio is approximately nine, and the principle of superposition holds, the maximum pressure-induced deflection can be related to the pressure via (2). The elastic modulus can then be calculated by plotting the maximum pressure-induced deflection versus pressure data and by finding the slope, which provides the best fitting of the data, via the linear regression method; the elastic modulus is linearly related to the slope of the maximum pressure-induced deflection versus pressure data via (2). The maximum pressure-induced deflection versus pressure data for six polysilicon films are shown in Fig. 5, where the prediction using the theory with the average $E$ of $93.5 \mathrm{GPa}$ is also indicated, and the slopes and elastic moduli calculated are given in Table I. The results show that the elastic modulus of polysilicon film is $93.5 \pm 2.6 \mathrm{GPa}$. It can be noted from (2) that

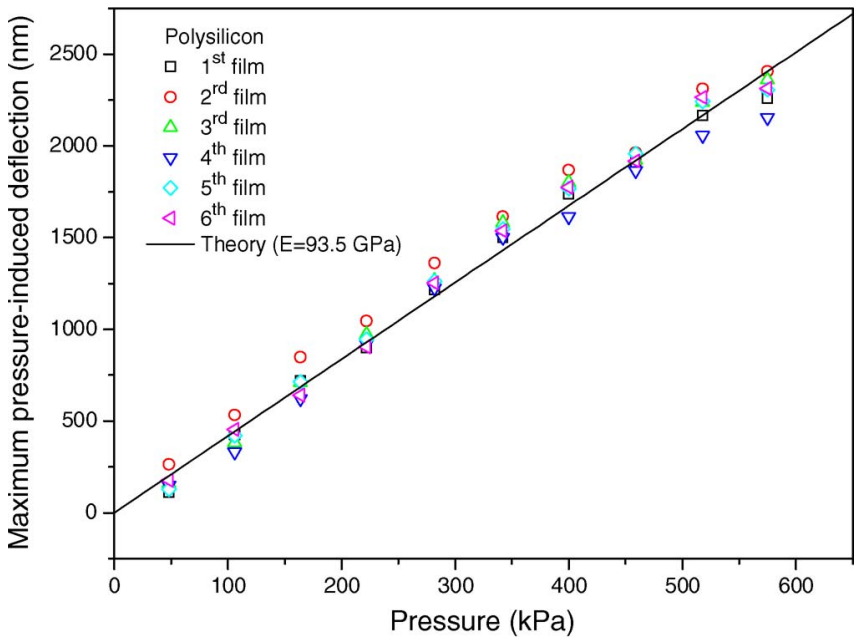

Fig. 5. Maximum pressure-induced deflection-pressure data of six polysilicon films obtained by the Twyman-Green interferometry, where the prediction from the analytic solution with the average $E$ of $93.5 \mathrm{GPa}$ is included.

the calculation of elastic modulus is very sensitive to the film geometry, so that it is important to measure the film dimension precisely. An optical microscope was used to measure the film thickness and width, which were found to be 10 and $340 \mu \mathrm{m}$, respectively. In the calculation of elastic modulus, the Poisson's ratio was assumed to be 0.25 . It has been reported, however, that the Poisson's ratio of polysilicon varies in the range of $0.15-0.3$, depending on the manufacturing process [6], [8]. The effect of the Poisson's ratio on the elastic modulus was examined in the range of $0.2-0.3$, and the result is given in Table II. The result reveals that the error, which originated from the uncertainty in the Poisson's ratio, is less than $\sim 5 \%$.

The FE analysis using a commercial software, ANSYS, was carried out to evaluate the elastic modulus. In the analysis, one polysilicon film was modeled with the symmetric boundary conditions because a plurality of actuators were arranged in parallel. The details used in the model are as follows: $1)$ isotropic and linear elastic materials $(\nu=0.25)$ and 2) plane strain condition (type of element used: solid82). Elastic modulus can be determined by comparing the maximum pressureinduced deflection versus pressure data obtained from both the experiment and the FE analysis. The procedures can be summarized as follows. First, the bulging process of the film is simulated with a guessed value of the elastic modulus, and the corresponding output is compared to the experimental result. If two results match within an acceptable level, then the guessed value is adopted as the elastic modulus of polysilicon film. Otherwise, the guessed value is modified, and the aforementioned procedures are repeated until a proper one is found. The average value of six polysilicon films was used to represent the experimental data, and the elastic modulus was calculated to be $107 \mathrm{GPa}$ (Table II). The effect of the Poisson's ratio was also investigated, and the result is given in Table II. It was found that the uncertainty in the Poisson's ratio can give rise to the error of $<\sim 5 \%$ in the elastic modulus. The transverse pressure-induced deflection profiles at the middle section under the pressure of $518 \mathrm{kPa}$, obtained from the experiment, the analytic solution, and the FE analysis, are compared in Fig. 6. The predictions 
TABLE I

Elastic Moduli of Six Polysilicon Films Calculated From the Thin-Plate Theory $[(2)(\nu=0.25)]$

\begin{tabular}{ccccccccc}
\hline Unit no. & 1 & 2 & 3 & 4 & 5 & 6 & Mean & SD \\
\hline Slope* & 4.15 & 4.13 & 4.30 & 3.99 & 4.27 & 4.25 & 4.18 & 0.11 \\
$(\mathrm{~nm} / \mathrm{kPa})$ & & & & & & & & \\
$\mathrm{E}(\mathrm{GPa})$ & 94.1 & 94.7 & 90.9 & 97.9 & 91.6 & 92.0 & 93.5 & 2.6 \\
\hline
\end{tabular}

* indicates the slope of the curve fitting the maximum pressure-induced deflection vs. pressure data.

TABLE II

Effect of Poisson's Ratio on the Elastic Modulus of POLYSILICON FILM

\begin{tabular}{ccc}
\hline \multirow{2}{*}{ Poisson's ratio } & \multicolumn{2}{c}{ Elastic modulus (GPa) } \\
\cline { 2 - 3 } & Thin plate theory & FE analysis \\
\hline 0.2 & 95.8 & 109.5 \\
0.25 & 93.5 & 107.0 \\
0.3 & 90.8 & 103.8 \\
\hline
\end{tabular}

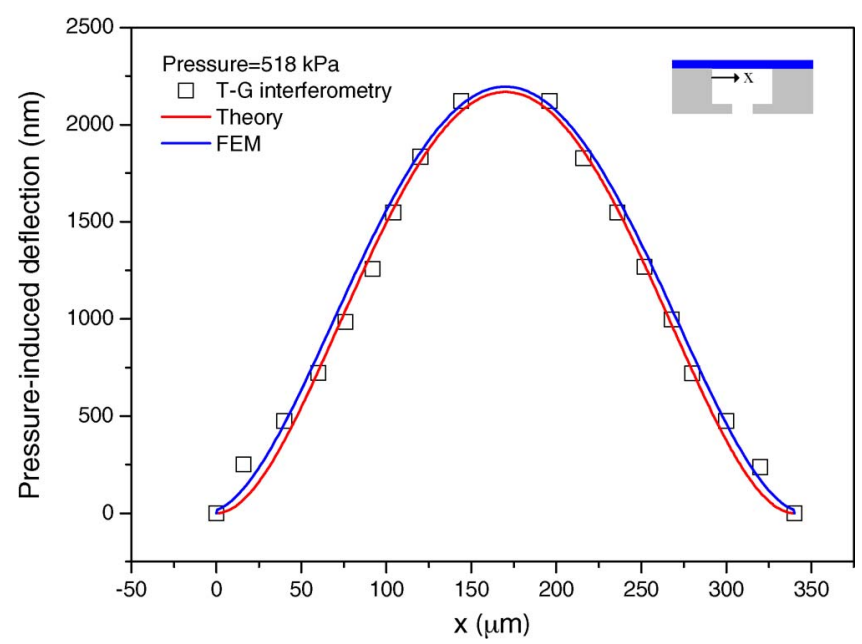

Fig. 6. Transverse pressure-induced deflection profiles at the middle section of the third polysilicon film under the pressure of $518 \mathrm{kPa}$ obtained by the Twyman-Green interferometry, thin-plate theory, and FE analysis.

from the analytic solution and the FE analysis appear to be in good agreement with the experimental result. It can be noted, however, that the analytic solution slightly underestimates the deflection, particularly around the film edges clamped. This fact is considered significant as the research goal moves to the measurement of the fracture strength since the maximum (fracture) stress $\sigma_{x x}$ occurs at the bottom of the edge clamped, and thus, the accurate stress calculation through the FE analysis is required.

\section{B. PZT Film}

The transverse deflection profiles at the middle sections of six consecutively arrayed PZT films are shown in Fig. 7; it should be noted that the setup for ESPI was identical to that in the

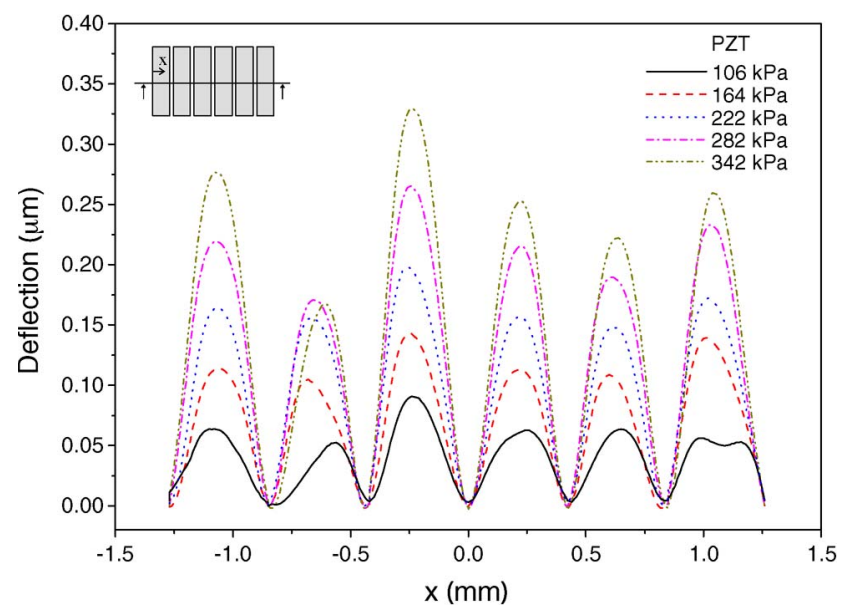

Fig. 7. Transverse deflection profiles at the middle sections of six PZT films obtained by ESPI.

comparative test. The deflection of films increases linearly with an increase in pressure, and their deflection shapes have analogy to those of polysilicon films except at low pressures; the second PZT film failed at $222 \mathrm{kPa}$, so there was no remarkable change in the deflection profile beyond $222 \mathrm{kPa}$. It can be noted, however, that the deflection profiles of six PZT films with the pressure are different to one another, unlike those of polysilicon films. Fig. 8 shows the height profiles of PZT films at the middle section. It was found that PZT films do not have uniform thicknesses along the width and that their configurations deposited are also slightly different to one another, and this nonuniformity in the film geometry makes the individual film deflect differently; it is known that the uniformity in the film geometry is hardly achieved as the film becomes thicker.

The FE analysis was used to evaluate the elastic modulus of PZT film, where the geometry of the film shown in Fig. 8 was taken into account separately; for the case that PZT film is uniformly deposited over the region entirely covering the ink chamber, the analytic solution derived from the rule of mixture formula can be available [18], but the specimens in this paper are not the case. In the analysis, one multilayer actuator, composed of polysilicon and PZT films, was modeled with the symmetric boundary conditions since a few tens of actuators were arrayed in parallel, and for simplicity, plane strain condition was assumed along the length direction. The details used in the model are as follows: 1) isotropic and linear elastic materials $\left(E_{\mathrm{Si}}=107 \mathrm{GPa}\right.$ and $\left.\nu_{\mathrm{Si}}=\nu_{\mathrm{PZT}}=0.25\right)$ and 

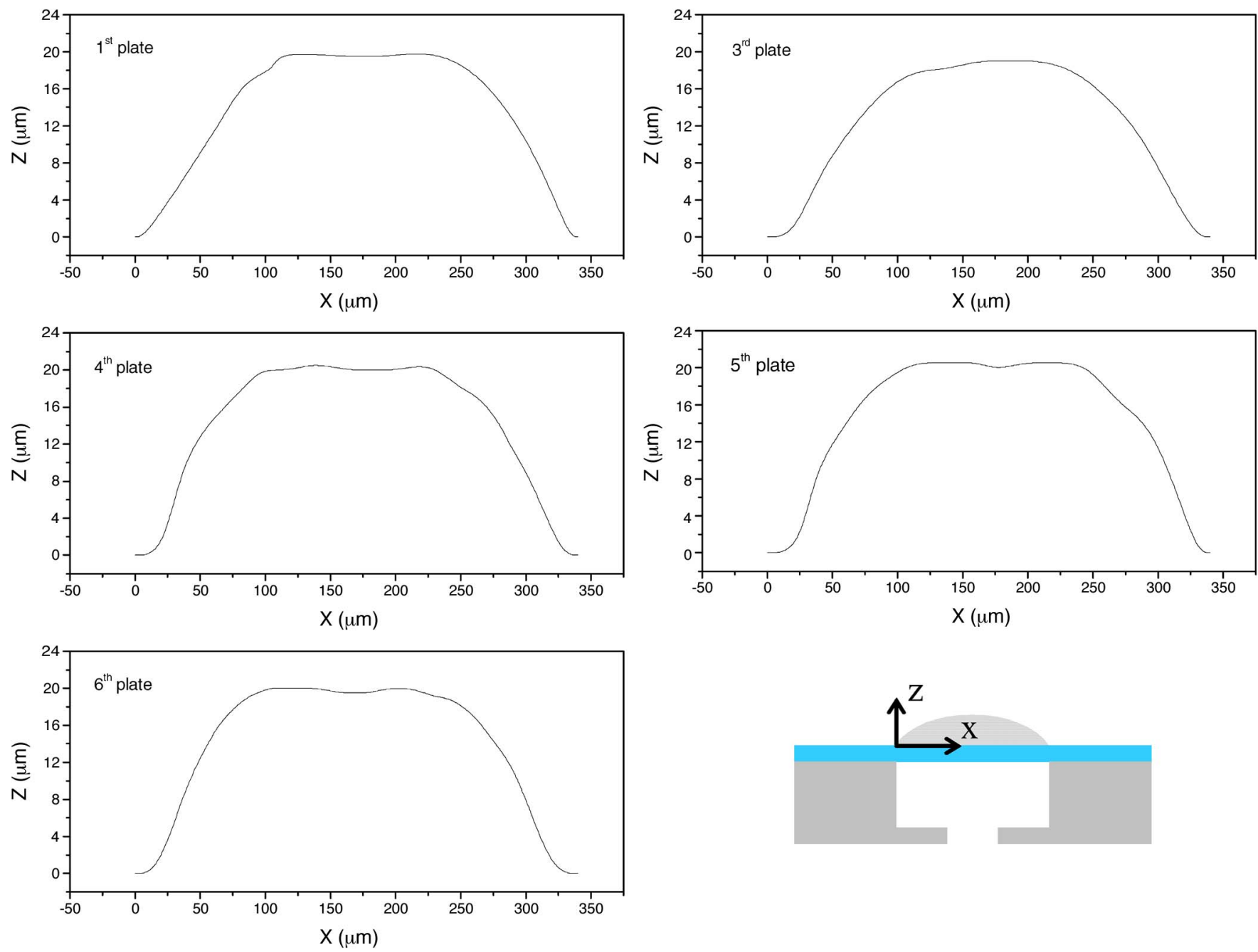

Fig. 8. Configuration of the middle cross sections of five PZT films.

2) plane strain condition (type of element used: solid82). The methodology to determine the elastic modulus is the same as that used in polysilicon film. The maximum deflection-pressure data of five PZT films are shown in Fig. 9, where the predictions from the FE analysis are indicated as the solid lines, and the elastic moduli calculated are given in Table III. The results reveal that the elastic modulus of PZT is $49.6 \pm 5.8 \mathrm{GPa}$.

Fig. 10 shows the optical micrographs of the fractured multilayer actuators (PZT specimens). In most of the fractured actuators, fracture topology was the separation of the films from the substrate, which ranged from the center to one end, and cracks were also observed at the ink chamber walls; sometimes, cracks initiated at the ink chamber walls without any fracture of the films. The bending at the edges of the film results in high local stress. The FE analysis results reveal that the maximum stress $\sigma_{x x}$ occurs at the bottom edges of the middle section; other stress components are considerably small compared to $\sigma_{x x}$. It is noted that, in the vicinity of the film ends, the stress distribution deviates from the plane strain model; the plane strain condition is approximated over the length of roughly $L-a$ around the middle section, where $L$ is the film length. Moreover, as shown in Fig. 8, the nonuniformity in the PZT film thickness becomes pronounced around the edges, and this intensifies the stress

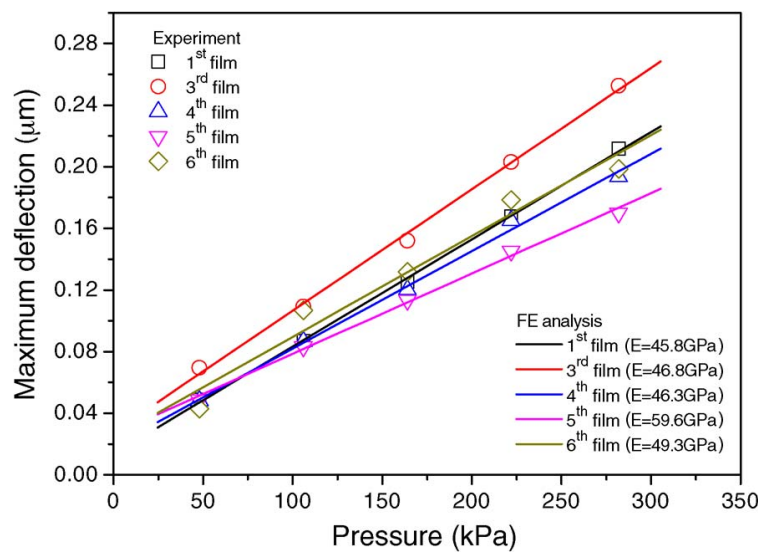

Fig. 9. Maximum deflection-pressure data of five PZT films obtained by ESPI and their predictions via the FE analysis.

concentration at the bottom edges of polysilicon film. Hence, during the bulging process, the maximum stress $\sigma_{x x}$ takes place at the bottom edges around the middle section, and this fact reasonably supports the fracture topology observed, where the breaking (or separation) of the films started from the same positions. 
TABLE III

Elastic Moduli of Five PZT Films CAlculated From the FE Analysis $(\nu=0.25)$

\begin{tabular}{lccccccc}
\hline Unit no. & 1 & 3 & 4 & 5 & 6 & Mean & SD \\
\hline E (GPa) & 45.8 & 46.8 & 46.3 & 59.6 & 49.3 & 49.6 & 5.8 \\
\hline
\end{tabular}

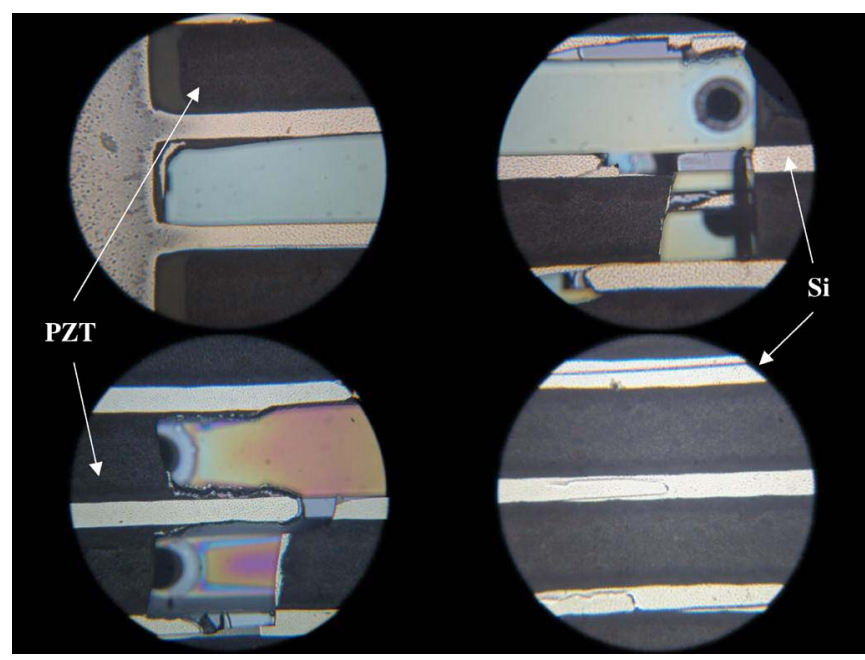

Fig. 10. Optical micrographs of the fractured multilayer actuators (PZT specimens).

\section{Uncertainty in the Measured Elastic Moduli}

Results on polysilicon film have shown that the elastic modulus varies from 95 to $190 \mathrm{GPa}$, depending on the test method and the manufacturing process [5]-[8]. This large variation in elastic modulus may be attributed to a combination of experimental uncertainty, microstructural difference, and lack of test data for statistical analysis; the difference in the used test methods was found to be unimportant as the results for tensile, static beam, resonant beam, and bulge tests are all widely scattered [8].

Differences in microstructure have been documented and are expected to have some effect on the elastic properties [5], [7]-[10]. For LPCVD polysilicon films, it has been reported that there is a critical temperature near $600{ }^{\circ} \mathrm{C}$, below which the deposited films are amorphous, and above which polycrystalline films are produced [6]-[8], [19]-[21]. The polycrystalline films have columnar grains, oriented perpendicular to the plane of the films, so they have a strong $\langle 110\rangle$ out-of-plane texture. Although the relative crystallographic orientation of the texture has been shown to depend on the deposition temperature [19]-[21], pressure [19], [21] and doping [21], the vast majority of microstructural studies on LPCVD polysilicon films have reported the presence of columnar grains and a strong $\langle 110\rangle$ out-of-plane texture [6]-[8]. Assuming a pure $\langle 110\rangle$ texture and using the Voigt-Reuss method [22], bounds for elastic constants of a defect-free polysilicon film have been calculated [8], [23]. The results yielded the elastic modulus of 162-171 GPa and the Poisson's ratio of $0.22-0.24$; the differences in the reference values for the stiffness matrix of single-crystalline silicon introduced a variation of several gigapascals in the calculated elastic modulus. It can be noted that the net effect of the $\langle 110\rangle$ texturing gives rise to just a small increase $(<\sim 3 \mathrm{GPa})$ in the elastic modulus. The effects of other texture orientations, i.e., $\langle 100\rangle$ and $\langle 111\rangle$, have also been examined [23]; the elastic moduli of the pure $\langle 100\rangle$ and $\langle 111\rangle$ textures were calculated to be 149.3 and $171.5 \mathrm{GPa}$, respectively. Their effects on the elastic modulus appear to be more significant compared to the $\langle 110\rangle$ texture but not crucial. Therefore, it can be concluded that variations of crystallographic texture in polysilicon may lead to differences in elastic modulus, but their effects are not crucial. At present, a value of $160-165 \mathrm{GPa}$ is generally accepted as the elastic modulus of polysilicon films with strongly $\langle 110\rangle$ textured microstructures [5].

When considering the aforementioned value for the elastic modulus of polysilicon films, the value measured in this paper is relatively small; a reduction of $\sim 30 \%$ is observed (the value of $\sim 110 \mathrm{GPa}$ from the FE analysis was adopted as the measured elastic modulus because the analytic solution slightly underestimated the film deflection behavior). This reduced elastic modulus may be attributed to experimental uncertainty. Using (2), the effects of inaccuracies in testing methodologies on the measured elastic modulus can be examined; $1 \%$ errors in the film thickness and width, the maximum deflection, and the applied pressure introduce errors of $3 \%, 4 \%, 1 \%$, and $1 \%$ in elastic modulus, respectively. For the Poisson's ratio $\nu$, it is dependent on the used value, and $1 \%$ error for $\nu=0.2$ gives rise to $0.1 \%$ error in elastic modulus (Table II). This error analysis result indicates that the measurement of elastic modulus through the bulge test is sensitive to the film geometry and deflection, specifically to the film thickness and width. However, it was found that although there may be some errors in measuring the film geometry and deflection, they are not large enough to account for the reduction of $30 \%$ in elastic modulus alone; the uniformity in the film geometry at acceptable level was evidenced through microscopic observation. The fact that experimental inaccuracy is not a main cause for the reduced elastic modulus can also be demonstrated by nanoindentation results (refer to Section III-D) in which the measured elastic modulus was $112.2 \pm 13.4 \mathrm{GPa}$, which is consistent with the bulge test result.

Elastic modulus is the macroscopic property controlled by the microscopic chemical bonding, so a change in the density of $\mathrm{Si}-\mathrm{Si}$ bonds per volume, which can originate from microdefects such as voids, microcracks, etc., would influence elastic modulus [7], [10], [24]. For LPCVD polysilicon films deposited at $620{ }^{\circ} \mathrm{C}$, subsequent annealing process increased the elastic modulus in the range of 8-11 GPa, depending on the annealing temperature [7]. It was pointed out that an increase in the density of $\mathrm{Si}-\mathrm{Si}$ bonds per volume, presumably due to the reduction of porosity, was the probable reason for enhancing elastic modulus. Huang and Spaepen [10] have carried out microtensile testing of vapor-deposited $\mathrm{Ag}, \mathrm{Al}$, and $\mathrm{Cu}$ thin films 


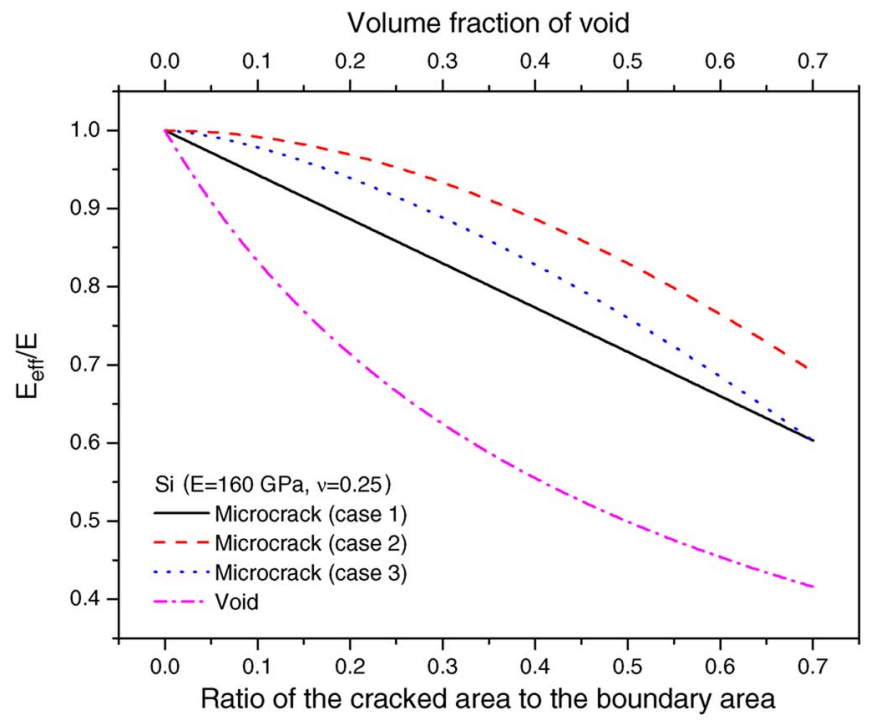

Fig. 11. Effects of voids and microcracks on the elastic modulus of polysilicon film; case 1,2, and 3 microcracks represent the fully detached grain boundary by slit-type crack, all boundaries detached to the same extent by slittype cracks, and all boundaries detached to the same extent by penny-shape cracks, respectively.

with thicknesses of several micrometers and found the reduction of $\sim 20 \%$ in elastic moduli compared to those calculated from single-crystal elastic constants. This reduction of elastic modulus was related to the incomplete cohesion at grain boundaries between columnar grains. In the deposited thin films, the boundaries between columnar grains are far from perfect as adjoining crystals are constrained by the substrate, and during deposition, the relaxation of boundary structures by diffusion is not complete. Hence, these imperfect grain boundaries can serve as likely sources of voids and microcracks, which will be ideally oriented to increase the film compliance. The effects of voids [25] and microcracks [26], [27] on elastic modulus were quantitatively analyzed; a detailed description can be found elsewhere [10]. In the analysis, voids were assumed to be randomly distributed and noninteracting, and spherical and microcracks were assumed to be randomly distributed at the grain boundaries of hexagonal columnar grains. The following three types of microcracks were exemplified for the convenience of one's understanding on the physical meaning involved: a fully detached grain boundary by slit-type crack (case 1), all boundaries detached to the same extent by slit-type cracks (case 2), and all boundaries detached to the same extent by penny-shape cracks (case 3 ). The analysis result is shown in Fig. 11, where elastic constants of a defect-free polysilicon film were assumed to be $E=160 \mathrm{GPa}$ and $\nu=0.25$. The results show that the values required for microdefects to introduce 10\%-20\% modulus reduction appear to be plausible based on the mechanism of deposition and the resulting microstructure. For example, a modulus reduction of $20 \%$ can be achieved if one in three grain boundaries is fully detached (case 1) or if all three grain boundaries are about half detached (case 2); note that there are three grain boundaries per grain. Therefore, it is thought that the reduced elastic modulus of polysilicon films measured in this paper is most likely attributed to the presence of microdefects like voids and microcracks at grain boundaries between columnar grains.
For PZT film, to the authors' knowledge, there have been no meaningful data to compare with our results; a few data are available, but they are bulk properties and are also highly varied, depending on manufacturing processes [28], [29]. Since the values measured by both bulge test and nanoindentation are consistent (refer to Section III-D for the nanoindentation result), the value of $\sim 49 \mathrm{GPa}$ can be regarded as the elastic modulus of PZT films. It can be noted that PZT film is intrinsically transversely isotropic, so that the assumption of isotropy may lead to some error. However, the consistency in the results obtained by the bulge test and the nanoindentation is thought to support that the degree of anisotropy in PZT film is not severe.

\section{Evaluation of Elastic Modulus by Nanoindentation}

Nanoindentations (Nanoindenter XP, MTS Systems Corporation, Oak ridge, $\mathrm{TN}$ ) were made on the cross sections of multilayer actuators, using a Berkovich indenter with a tip radius of $50 \mathrm{~nm}$. Samples were prepared by cutting multilayer actuators and polishing their cut sections up to a $0.05-\mu \mathrm{m}$ finish. The operating conditions of the nanoindenter were the following: 1) continuous stiffness mode; 2) drift rate $=0.05 \mathrm{~nm} / \mathrm{s}$; 3) Poisson's ratio $=0.25$; and 4) indentation depth $=500 \mathrm{~nm}$ (for both polysilicon and PZT films, the elastic moduli measured as a function of indentation depth were found to be nearly saturated beyond $500 \mathrm{~nm}$ ). Three indentations were made in each sample, and the number of indentations for polysilicon and PZT films were 18 and 15, respectively. The result revealed that the measured values are $112.2 \pm 13.4 \mathrm{GPa}$ for polysilicon and $49.0 \pm 15.2 \mathrm{GPa}$ for PZT, which are consistent with those obtained from the bulge test.

\section{CONCLUSION}

Deformation behaviors and elastic moduli of multilayered films, composed of polysilicon and PZT, in piezoelectric inkjet head were mechanically characterized by bulge testing commercial inkjet heads directly, where a layer-by-layer approach was adopted to characterize the elastic behavior of individual film. The corresponding deflections of films were measured by full-field optical measurement techniques, depending on the optical surface conditions of films, i.e., Twyman-Green interferometry for polysilicon film and ESPI for PZT film. The bulging process of samples could be described well via a thin-plate theory and FE analysis, and their predictions were compared with the experimental data to estimate the elastic moduli of both films. Here, the effect of initial deflection that originated from the fabrication process could be properly treated using a principle of superposition. The average elastic moduli of polysilicon and PZT films were calculated to be $\sim 110$ and $\sim 49 \mathrm{GPa}$, respectively, and these values were found to be consistent with the nanoindentation results. It was noted that, for polysilicon films, $\sim 30 \%$ reduction in elastic modulus, compared to that calculated from a probabilistic model of texture, was observed. This reduction of elastic modulus appears to be related to the microdefects, such as voids and microcracks, at grain boundaries between columnar grains. 


\section{ACKNOWLEDGMENT}

The authors would like to thank Dr. S. J. Ham for providing test specimens, Dr. T. S. Park and Dr. S. Y. Yang for helping construct the bulge testing system, and Dr. T. S. Byun for his technical review and thoughtful comments.

\section{REFERENCES}

[1] Epson press release. [Online]. Available: http://www.epson.co.jp/e/ newsroom/2007/news_20070327.htm

[2] Y. Zhou, "Recent advances in highly durable piezoelectric inkjet print head technology," in Proc. Int. Conf. Digital Printing Technol., 2004, pp. 855-858.

[3] A. S. Yang, J. C. Yang, and M. C. Hong, "Droplet ejection study of a Picojet printhead," J. Micromech. Microeng., vol. 16, no. 1, pp. 180-188, Jan. 2006.

[4] Y. S. Chen, Y. L. Huang, C. H. Kuo, and S. H. Chang, "Investigation of design parameters for droplet generators driven by piezoelectric actuators," Int. J. Mech. Sci., vol. 49, no. 6, pp. 733-740, Jun. 2007.

[5] K. J. Hemker and W. N. Sharpe, Jr., "Microscale characterization of mechanical properties," Annu. Rev. Mater. Res., vol. 37, pp. 93-126, 2007.

[6] W. N. Sharpe, Jr., K. M. Jackson, K. J. Hemker, and Z. Xie, "Effect of specimen size on Young's modulus and fracture strength of polysilicon," J. Microelectromech. Syst., vol. 10, no. 3, pp. 317-326, Sep. 2001.

[7] D. Maier-Schneider, A. Koprululu, S. B. Holm, and E. Obermeier, "Elastic properties and microstructure of LPCVD polysilicon films," J. Micromech. Microeng., vol. 6, no. 4, pp. 436-446, Dec. 1996.

[8] S. Jayaraman, R. L. Edwards, and K. J. Hemker, "Relating mechanical testing and microstructural features of polysilicon thin films," J. Mater. Res., vol. 14, no. 3, pp. 688-697, Mar. 1999.

[9] Y. Xiang, X. Chen, and J. J. Vlassak, "The mechanical properties of electroplated $\mathrm{Cu}$ thin films measured by means of the bulge test technique," in Proc. Mater. Res. Soc. Symp., 2002, vol. 695, pp. L4.9.1-L4.9.6.

[10] H. Huang and F. Spaepen, "Tensile testing of free-standing $\mathrm{Cu}, \mathrm{Ag}$ and $\mathrm{Al}$ thin films and Ag/Cu multilayers," Acta Mater., vol. 48, no. 12, pp. 32613269,2000

[11] S. Timoshenko and S. Woinowsky-Krieger, Theory of Plates and Shells, 2nd ed. New York: McGraw-Hill, 1959.

[12] Y. Xiang, X. Chen, and J. J. Vlassak, "Plane-strain bulge test for thin films," J. Mater. Res., vol. 20, no. 9, pp. 2360-2370, Sep. 2005.

[13] A. Bosseboeuf and S. Petitgrand, "Characterization of the static and dynamic behaviour of $\mathrm{M}(\mathrm{O}) \mathrm{EMS}$ by optical techniques: Status and trends," J. Micromech. Microeng., vol. 13, no. 4, pp. S23-S33, Jul. 2003.

[14] D. Post, B. Han, and P. Ifju, High Sensitivity Moire: Experimental Analysis for Mechanics and Materials. New York: Springer-Verlag, 1994.

[15] S. Y. Yang, Y. D. Jeon, S. B. Lee, and K. W. Paik, "Solder reflow process induced residual warpage measurement and its influence on reliability of flip-chip electronic packages," Microelectron. Reliab., vol. 46, no. 2-4, pp. 512-522, Feb.-Apr. 2006.

[16] Interferometry, optical metrology. [Online]. Available: http://optics.tugraz.ac.at

[17] R. C. Hibbeler, Mechanics of Materials. Englewood Cliffs, NJ: PrenticeHall, 1994.

[18] A. Karimi, O. R. Shojaei, T. Kruml, and J. L. Martin, "Characterization of TiN thin films using the bulge test and the nanoindentation technique," Thin Solid Films, vol. 308/309, pp. 334-339, Oct. 1997.

[19] T. I. Kamins, "Structure and properties of LPCVD silicon films," J. Electrochem. Soc., vol. 127, no. 3, pp. 686-690, Mar. 1980.

[20] N. Nagasima and N. Kubota, "Structures of Si films chemically vapordeposited on amorphous $\mathrm{SiO}_{2}$ substrates," Jpn. J. Appl. Phys., vol. 14, no. 8, pp. 1105-1112, Aug. 1975.

[21] P. Joubert, M. Sarret, L. Haji, L. Hamedi, and B. Loisel, "Pressure dependence of in situ boron-doped silicon films prepared by low-pressure chemical vapor deposition. I. Microstructure," J. Appl. Phys., vol. 66, no. 10 , pp. 4806-4811, Nov. 1989.

[22] R. Hill, "The elastic behavior of a crystalline aggregate," Proc. Phys. Soc. $A$, vol. 65 , pp. $349-354,1952$

[23] W. A. Brantley, "Calculated elastic constants for stress problems associated with semiconductor devices," J. Appl. Phys., vol. 44, no. 1, pp. 534535, Jan. 1973.

[24] W. N. Sharpe, Jr., J. Pulskamp, B. G. Mendis, C. Eberl, D. S. Gianola et al. "Tensile stress-strain curves of gold film,"Proc. ASME Int. Mech. Eng. Congr. Expo., 2006, pp. 1-8. 13290.
[25] J. D. Eshelby, "The determination of the elastic field of an ellipsoidal inclusion, and related problems," Proc. R. Soc. Lond. A, Math. Phys. Sci. vol. 241, no. 1226, pp. 376-396, Aug. 1957.

[26] B. Budiansky and R. J. O'Connell, "Elastic moduli of a cracked solid," Int. J. Solids Struct., vol. 12, pp. 81-97, 1976.

[27] N. Laws and J. R. Brockenbrough, "The effect of micro-crack systems on the loss of stiffness of brittle solids," Int. J. Solids Struct., vol. 23, no. 9, pp. 1247-1268, 1987.

[28] J. Lu, Y. Zhang, T. Kobayashi, R. Maeda, and T. Mihara, "Preparation and characterization of wafer scale lead zirconate titanate film for MEMS application," Sens. Actuators A, Phys., vol. 139, no. 1/2, pp. 152-157, Sep. 2007.

[29] K. Yamashita, H. Nishimoto, and M. Okuyama, "Diaphragm deflection control of piezoelectric ultrasonic microsensors for sensitivity improvement," Sens. Actuators A, Phys., vol. 139, no. 1/2, pp. 118-123, Sep. 2007.

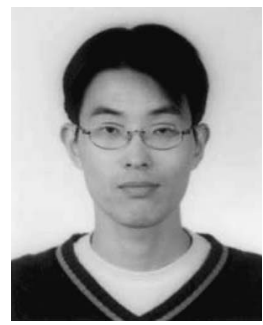

Seong-Gu Hong received the B.S., M.S., and Ph.D degrees from Korea Advanced Institute of Science and Technology, Daejeon, Korea, in 1997, 1999, and 2005 , respectively.

$\mathrm{He}$ is currently a Research Assistant Professor with the Department of Materials Science and Engineering, Pohang University of Science and Technology, Pohang, Korea. His research interests include mechanical reliability and durability evaluation of miniature applications, such as ceramic coatings in nuclear fuel systems and thin films in MEMS

applications.

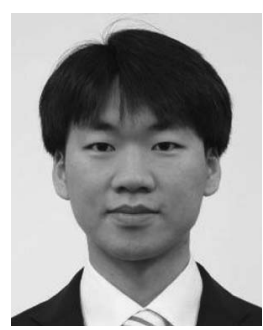

Minho Kim received the B.S. and M.S. degrees from Korea Advanced Institute of Science and Technology (KAIST), Daejeon, Korea, in 2002 and 2004, respectively. He is currently working toward the Ph.D. degree in the Department of Mechanical Engineering, KAIST.

His research interests include material characterization and structural analysis of thin films in electronic packaging systems using optical measurement techniques.

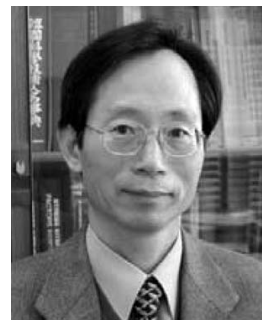

Soon-Bok Lee (S'77-M'80) received the Ph.D. degree from Stanford University, Stanford, CA, in 1980.

He is currently a Professor with the Department of Mechanical Engineering, Korea Advanced Institute of Science and Technology, Daejeon, Korea. He is a strong supporter of reliability through research, education, government regulation, society activities, and international cooperation and conferences. His technical interests include material characterization of thin film, reliability in microelectronics packaging, fatigue, and fracture mechanics.

Dr. Lee is a member of the American Society of Mechanical Engineers. He served as the Chairman of the National Reliability Committee and helped set up the national reliability assessment codes and system in Korea. He also served as the General Chairman of the IEEE CPMT-sponsored 3rd EMAP (Electronics Materials and Packaging) Conference in 2001. He organized the International Conference of Experimental Mechanics (ICEM2006) as the General Chairman.

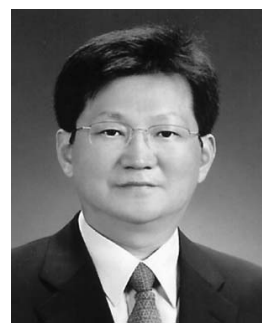

Chong Soo Lee received the B.S. and M.S. degrees from Seoul National University, Seoul, Korea, in 1979 and 1981, respectively, and the Ph.D. degree from the Polytechnic Institute of New York (now Polytechnic University), Brooklyn, in 1985, all in metallurgical engineering.

$\mathrm{He}$ is currently a Professor with the Department of Materials Science and Engineering, Pohang University of Science and Technology, Pohang, Korea. His research interests include fatigue life prediction and high-temperature formability of various metallic 